\title{
Detección precoz y manejo de la enfermedad de Kawasaki: la inadvertida enfermedad de Kawasaki incompleta
}

\author{
Ana Isabel Rabadán Velasco ${ }^{a}$, Aránzazu Recio Linares ${ }^{\mathrm{b}}$, Inmaculada Cabello García ${ }^{\mathrm{b}}$, \\ Esther Crespo Rupérez ${ }^{c}$
}

\begin{abstract}
a Médico Interno Residente de Medicina Familiar y Comunitaria, Centro de Salud de Sillería (Toledo)

b Médico Interno Residente de Pediatría y otras Áreas específicas, Hospital Virgen de la Salud (Toledo)

- Médico Adjunto. Servicio de Urgencias de Pediatría, Hospital Virgen de la Salud (Toledo)
\end{abstract}

Correspondencia: Ana Isabel Rabadán Velasco. Plaza de San Justo 2, $2^{\circ}$ E. 45001 , Toledo, España.

Correo electrónico: aranaira@ gmail.com

Recibido el 7 de noviembre de 2012.

Aceptado para su publicación el 25 de agosto de 2012.

\begin{abstract}
RESUMEN
La enfermedad de Kawasaki (EK) incompleta supone el no cumplimiento de todos los criterios diagnósticos del Kawasaki completo o síndrome mucocutáneo linfonodular, siendo necesario un hallazgo de fiebre de al menos cinco días de evolución y dos o tres criterios clínicos, así como una serie de criterios de laboratorio. Las complicaciones no difieren del síndrome completo, por lo que estos pacientes tienen el mismo riesgo de desarrollar alteraciones cardiovasculares tales como aneurismas y arteritis coronarias, disminución de la fracción de eyección del ventrículo izquierdo o derrame pericárdico. Diversos estudios constatan un diagnóstico ineficaz de la EK incompleta, y consecuentemente una mayor frecuencia de alteraciones ecocardiográficas. Se presenta el caso de un paciente con fiebre de cinco días de evolución y exantema generalizado que posteriormente presenta dolor testicular, eritema perineal, enantema e inyección conjuntival que finalmente fue diagnosticado de EK incompleta.
\end{abstract}

Palabras Clave: Síndrome Mucocutáneo Linfonodular, Arteritis, Exantema.

Early detection and management of Kawasaki disease: the undetected incomplete Kawasaki disease

Incomplete Kawasaki disease (KD) means the non-compliance with all diagnostic criteria of complete Kawasaki, or mucocutaneous lymph node syndrome, with the presence of fever lasting at least five days and two or three clinical criteria, as well as a series of laboratory criteria. Complications do not differ from the complete syndrome, which is why these patients are subject to the same risk of developing cardiovascular disorders such as aneurysms, coronary arteritis, decreased ejection fraction in the left ventricle or pericardial effusion. Several studies have shown an inefficient diagnosis of incomplete KD and consequently, a greater frequency of echocardiographic alterations. The case of a patient with a fever lasting five days and a generalized rash is reported, who subsequently develops testicular pain, perineal erythema, enanthema and a bloodshot appearance, who was finally diagnosed with incomplete KD.

Key words: Mucocutaneous Lymph Node Syndrome, Arteritis, Exanthema.

\section{INTRODUCCIÓN}

La enfermedad de Kawasaki (EK) es una vasculitis sistémica que afecta a vasos de mediano calibre, fundamentalmente a las arterias coronarias ${ }^{1}$. Es más frecuente en niños menores de 4-5 años y predomina en el sexo masculino (varón-mujer 1,4:1).

La etiología es desconocida, pero las similitudes que comparte con el Síndrome del Shock Tóxico Estafilocócico y el Estreptocócico sugieren la participación de un superantígeno en la patogenia de esta enfermedad. La EK fue descubierta por el Dr. Tomisaku Kawasaki en el año 1961 en Japón, donde se registra la mayor incidencia de esta enfermedad (108/100.000 niños $<5$ años por año) $)^{2}$.

Existen dos formas de presentación de la enfermedad que se resumen en la tabla $1^{3}$. La American Heart Association (AHA), y la American Academy of Pediatrics (AAP) han establecido una guía para el diagnóstico y manejo de la EK, donde se incluye un algoritmo (figura 1$)^{4}$. 


\section{OBSERVACIONES CLÍNICAS}

Paciente varón de 3 años de edad que es remitido por su pediatra al Servicio de Urgencias del
Hospital Virgen de la Salud de Toledo por presentar un exantema escarlatiniforme generalizado de dos días de evolución, acompañado de fiebre de hasta $38,6^{\circ} \mathrm{C}$. No presentaba antecedentes personales

\begin{tabular}{|l|l|}
\hline \multicolumn{1}{|c|}{ Enfermedad de Kawasaki completa } & \multicolumn{1}{c|}{ Enfermedad de Kawasaki incompleta } \\
\hline $\begin{array}{l}\text { Fiebre de al menos } 5 \text { días de evolución }+ \text { al menos 4 de los } \\
\text { siguientes criterios clínicos: }\end{array}$ & $\begin{array}{l}\text { Fiebre de al menos } 5 \text { días }+2 \text { ó } 3 \text { criterios clínicos }+ \text { PCR }>30 \\
\mathrm{mg} / \mathrm{l} \text { ó VSG }>40 \mathrm{~mm} / \mathrm{h}+\text { al menos } 3 \text { de los siguientes criterios } \\
\text { de laboratorio: }\end{array}$ \\
\hline$-\quad$ Inyección conjuntival bilateral no exudativa. & $-\quad$ Albúmina $\leq 3 \mathrm{~g} / \mathrm{dll}$. \\
\hline$-\quad \begin{array}{l}\text { Alteraciones de la mucosa bucal: lengua aframbuesada, } \\
\text { enantema, labios fisurados. }\end{array}$ & $-\quad$ Anemia normocítica y normocrómica para la edad. \\
\hline$-\quad$ Exantema polimorfo. & $-\quad$ Elevación de GPT. \\
\hline$-\quad$ Lesión palmoplantar: eritema, descamación. & $-\quad$ Plaquetas $>450.000 /$ mm3 después de 7 días. \\
\hline$-\quad$ Adenopatía cervical $>1,5 \mathrm{~cm}$. & $-\quad$ Leucocitos $>15.000 /$ mm3. \\
\hline & $-\quad 10$ células / campo en el sedimento de orina. \\
\hline
\end{tabular}

Tabla 1. Criterios diagnósticos de la enfermedad de Kawasaki completa e incompleta.

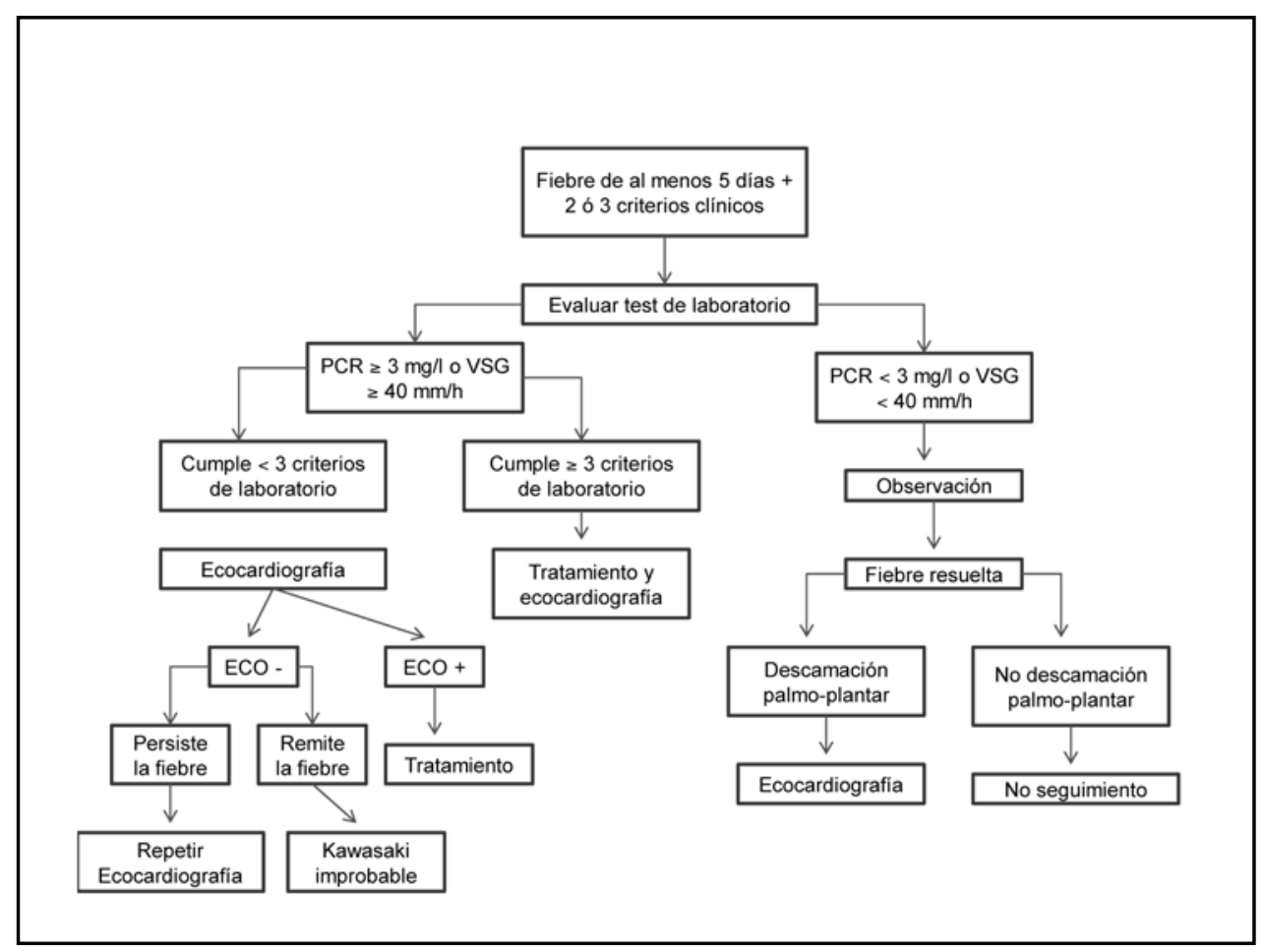

Figura 1. Algoritmo para el diagnóstico y manejo de la enfermedad de Kawasaki según la American Heart Association. 
de interés. En la exploración física se objetivó un exantema maculopapuloso no rasposo y una orofaringe hiperémica con exudado blanquecino (figura 2). Se realizó la prueba de detección rápida de antígeno de estreptococo, que resultó negativa. El paciente fue dado de alta con el diagnóstico de exantema inespecífico.

Al día siguiente regresó al Servicio de Urgencias por dolor testicular y persistencia de la fiebre. Se observó leve eritema en región perineal. Se decidió alta con antitérmicos y analgesia, indicando volver si no cedía la fiebre. El quinto día de evolución el paciente regresó a Urgencias con marcada

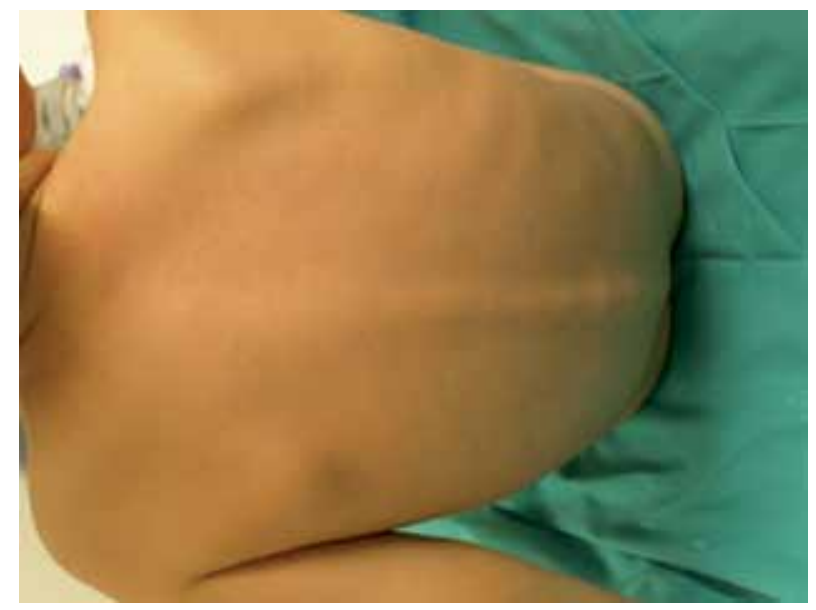

Figura 2. Exantema escarlatiniforme en tronco. irritabilidad, acompañado de intenso eritema en región perineal, labios agrietados y enantema en paladar (figuras 3 y 4 ).

Al no cumplir los criterios diagnósticos de linfadenopatía cervical unilateral ni afectación de extremidades, se decidió realizar una analítica completa con el objetivo de descartar una EK incompleta. En los resultados obtenidos destacó: PCR 90 mg/l, VSG 55 mm, albúmina 2,6 g/dl, GPT $175 \mathrm{mU} / \mathrm{ml}$ y piuria en el sedimento de orina. Se decidió ingreso del paciente por cumplir criterios de EK incompleta. Al inicio del ingreso se realizó ecocardiograma donde se evidenciaron arterias

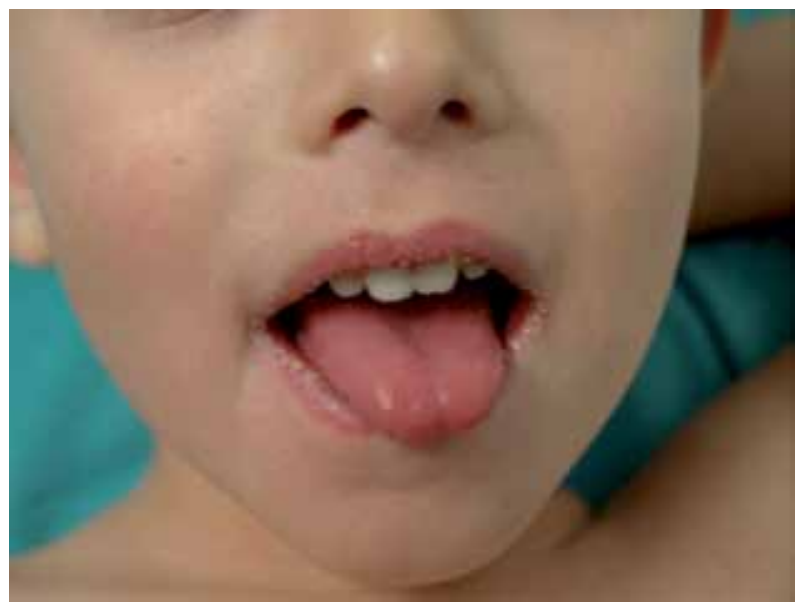

Figura 3. Lengua fisurada y labios agrietados.

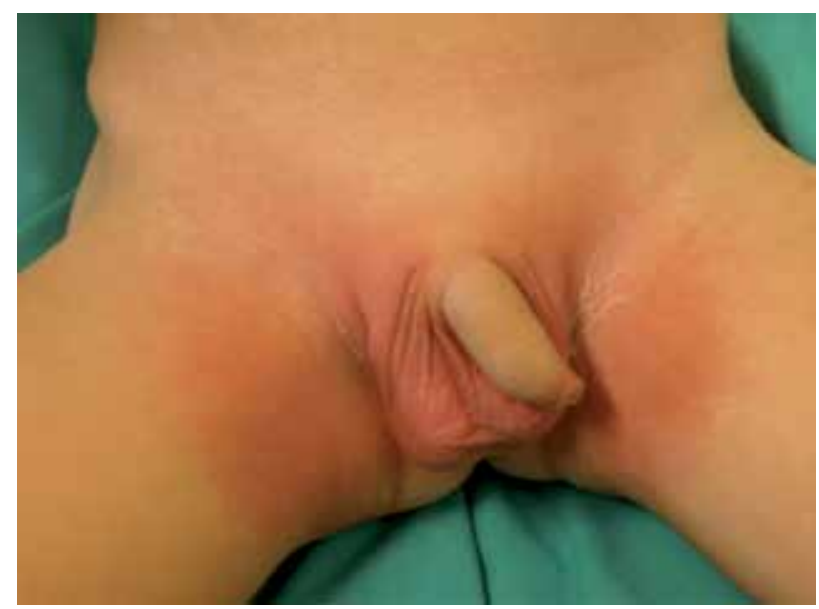

Figura 4. Eritema genital. 
coronarias normales y se inició tratamiento con gammaglobulina a dosis de $2 \mathrm{~g} / \mathrm{kg}$ en infusión durante $12 \mathrm{~h}$ junto con ácido acetilsalicílico (AAS) a dosis de 1,5 g/24 h en 4 dosis. Durante los tres primeros días del ingreso presentó picos febriles, se realizó frotis faringoamigdalar y serologías para virus de Epstein-Barr, citomegalovirus y Rickettsia, que fueron negativos. A las 72 horas afebril, se decidió alta con dosis de AAS a $50 \mathrm{mg} / 24 \mathrm{~h}$, control ecocardiográfico al mes siguiente y revisión por el Servicio de Dermatología, que objetivó a los 13 días del inicio del cuadro descamación en colgajos en dedos de las manos y alguno del pie, junto con descamación furfurácea en glúteos.

\section{COMENTARIOS}

La variabilidad de los signos y síntomas clínicos de la EK obligan a realizar un amplio diagnóstico diferencial, que incluya otros procesos infecciosos e inflamatorios como son la sarcoidosis, la panarteritis nodosa e incluso cuadros reumatológicos.

Existen 2 situaciones en las que será muy importante reconocer una EK incompleta (10\% del total de $\mathrm{EK})^{5}$. Estas circunstancias serían:

- Menor de 6 meses con fiebre inexplicable mayor o igual a 7 días (en el grupo de niños menores de 1 año se encuentra una mayor frecuencia de EK incompleto y de afectación coronaria) ${ }^{6}$.

- Cualquier edad con fiebre inexplicable de más de 5 días y menos de 3 criterios diagnósticos, si cumple criterios analíticos, como corresponde al caso expuesto.

Será básico, por tanto, recordar que la fiebre es el primer síntoma, el más constante y sobre el que gira el diagnóstico y pronóstico, y que una erupción a nivel perineal debe hacer sospechar la enfermedad.

Otro síntoma importante es un estado de irritabilidad marcada. Se trata de un síntoma vinculado a una hipoperfusión cerebral transitoria, observada en la fase aguda de la enfermedad consecuencia de la propia vasculitis.

Nuestro paciente realizó el tratamiento recomendado antes de los 7 días de inicio de la enfermedad con AAS y una dosis única de gammaglobulina hiperinmune. La gammaglobulina actúa neutralizando los anticuerpos, bloqueando los receptores Fc de las inmunoglobulinas y favoreciendo el aclaramiento de las citocinas. Por su parte, la utilización del AAS se ha basado en su capacidad para disminuir la duración de la fiebre, la normalización de los reactantes de fase aguda y la mejoría de la función miocárdica ${ }^{7}$.

Consideramos este caso relevante clínicamente, ya que el diagnóstico de la enfermedad de Kawasaki se basa en criterios clínicos y será en muchas ocasiones la pericia del médico la responsable de captar muchas de sus sutiles manifestaciones iniciales y sospechar con ello la enfermedad.

\section{BIBLIOGRAFÍA}

1. Sudo D, Monobe $\mathrm{Y}$, Yashiro $\mathrm{M}$, Mieno MN, Uehara R, Tsuchiya $\mathrm{K}$ et al. Coronary artery lesions of incomplete Kawasaki Disease: a nationwide survey in Japan. Eur J Pediatr. 2012; 171(4):651-6.

2. Rowley AH, Shulman ST. Enfermedad de Kawasaki. En: Kliegman RM, Behrman RE, Jenson HB, Stanton BF, editores. Nelson Tratado de Pediatría Vol. 1. $18^{\mathrm{a}}$ ed. Madrid: Elsevier; 2009.p.1036-42.

3. Ybarra Zavala M, Guerrero Fernández J, Del Castillo Martín F. Enfermedades Exantemáticas. En: Guerrero Fernández J, Ruiz Domínguez JA, Menéndez Suso JJ, Barrios Tascón A, editores. Manual de Diagnóstico y Terapéutica en Pediatría. $5^{\text {a }}$ ed. Madrid: Publimed; 2009.p.663-85.

4. Sundel R. Incomplete (atypical) Kawasaki disease. [Monografía en Internet]. Walthman (MA): UpToDate; 2012. [actualizada 18 de junio de 2012; acceso 30 de Julio de 2012]. Disponible en: http://www.uptodate.com.

5. Joffe A, Kabani A, Jadavji T. Atypical and complicated Kawasaki disease in infants. Do we need criteria? West $\mathrm{J}$ Med. 1995; 162(4):322-7.

6. Liu HC, Lo CW, Hwang B, Lee PC. Clinical manifestations vary with different age spectrums in infants with Kawasaki Disease. ScientificWorldJournal. 2012; 2012: 210382. doi: 10/11000/2012/210382.

7. Pucci A, Martino S, Tibaldi M, Bartoloni G. Incomplete and atypical Kawasaki disease: a clinicopathologic paradox at high risk of sudden and unexpected infants death. Pediatr Cardiol. 2012; 33(5):802-5. 